\title{
Generalized integral inequalities for discontinuous functions with two independent variables and their applications
}

\section{Yuzhen $\mathrm{Mi}^{*}$}

\section{*Correspondence:}

miyuzhen2009@126.com

Department of Mathematics,

Zhanjiang Normal University,

Zhanjiang, Guangdong 524048

China

\begin{abstract}
This paper investigates integral inequalities for discontinuous functions with two independent variables involving two nonlinear terms. We do not require that $\omega(u)$ is in the class $\wp$ or the class $\boldsymbol{J}$ in Gallo and Piccirllo's paper (Nonlinear Stud. 19:1 15-126, 2012). My main results can be applied to generalize Borysenko and lovane's results (Nonlinear Anal., Theory Methods Appl. 66:2190-2230, 2007) and to give results similar to Gallo-Piccirllo's. Examples to show the bounds of solutions of a partial differential equation with impulsive terms are also given, which cannot be estimated by Gallo and Piccirllo's results.
\end{abstract}

MSC: 26D15; 26D20

Keywords: integral inequalities; discontinuous functions; impulse; nonlinear

\section{Introduction}

Integral inequalities and their various linear and nonlinear generalizations involving continuous or discontinuous functions play very important roles in investigating different qualitative characteristics of solutions for differential equations, partial differential equations and impulsive differential equations such as existence, uniqueness, continuation, boundedness, continuous dependence of parameters, stability, and attraction. The literature on inequalities for continuous functions and their applications is vast (see [1-11]). In the one-dimensional case, all the main results in the theory of integral inequalities for continuous functions are almost based on the solvability of Chaplygin's problem [6] for the integral inequality

$$
u(x) \leq \varphi(x)+\int_{x_{0}}^{x} \Gamma(x, s, u(s)) d s
$$

Recently, more attention has been paid to generalizations of Gronwall-Bellman's results for discontinuous functions and their applications (see [12-27]). One of the important things is that Samoilenko and Perestyuk [26] studied the following inequality:

$$
u(x) \leq c+\int_{x_{0}}^{x} f(s) u(s) d s+\sum_{x_{0}<x_{i}<x} \beta_{i} u\left(x_{i}-0\right)
$$

\section{Springer}

( $2014 \mathrm{Mi}$; licensee Springer. This is an Open Access article distributed under the terms of the Creative Commons Attribution License (http://creativecommons.org/licenses/by/2.0), which permits unrestricted use, distribution, and reproduction in any medium, provided the original work is properly cited. 
for the nonnegative piecewise continuous function $u(x)$, where $c, \beta_{i}$ are nonnegative constants, $f(x)$ is a positive function, and $x_{i}$ are the first kind discontinuity points of the function $u(x)$. Then Borysenko [14] investigated integral inequalities with two independent variables,

$$
\begin{aligned}
u(x, y) \leq & a(x, y)+\int_{x_{0}}^{x} \int_{y_{0}}^{y} \tau(s, t) u(s, t) d s d t \\
& +\sum_{\left(x_{0}, y_{0}\right)<\left(x_{i}, y_{i}\right)<(x, y)} \beta_{i} u\left(x_{i}-0, y_{i}-0\right) .
\end{aligned}
$$

Here $u(x, y)$ is an unknown nonnegative continuous function with the exception of the points $\left(x_{i}, y_{i}\right)$ where there is a finite jump: $u\left(x_{i}-0, y_{i}-0\right) \neq u\left(x_{i}+0, y_{i}+0\right), i=1,2, \ldots$

In 2007, Borysenko and Iovane [16] considered the following inequalities:

$$
\begin{aligned}
& u(x, y) \leq a(x, y)+\int_{x_{0}}^{x} \int_{y_{o}}^{y} \tau(s, t) u(s, t) d s d t \\
& +\sum_{\left(x_{0}, y_{0}\right)<\left(x_{i}, y_{i}\right)<(x, y)} \beta_{i} u^{m}\left(x_{i}-0, y_{i}-0\right), \quad m>0 \text {, } \\
& u(x, y) \leq a(x, y)+\int_{x_{0}}^{x} \int_{y_{o}}^{y} \tau(s, t) u^{m}(s, t) d s d t \\
& +\sum_{\left(x_{0}, y_{0}\right)<\left(x_{i}, y_{i}\right)<(x, y)} \beta_{i} u^{m}\left(x_{i}-0, y_{i}-0\right), \quad m>0, \\
& u(x, y) \leq a(x, y)+q(x, y) \int_{x_{0}}^{x} \int_{y_{o}}^{y} \tau(s, t) u^{m}(\sigma(s), \sigma(t)) d s d t \\
& +\sum_{\left(x_{0}, y_{0}\right)<\left(x_{i}, y_{i}\right)<(x, y)} \beta_{i} u^{m}\left(x_{i}-0, y_{i}-0\right), \quad m>0 \text {. }
\end{aligned}
$$

Later, Gallo and Piccirllo [24] studied the following inequalities:

$$
\begin{aligned}
u(x, y) \leq & a(x, y)+q(x, y) \int_{x_{0}}^{x} \int_{y_{o}}^{y} \tau(s, t) \omega(u(\sigma(s), \tau(t))) d s d t \\
& +\sum_{\left(x_{0}, y_{0}\right)<\left(x_{i}, y_{i}\right)<(x, y)} \beta_{i} u^{m}\left(x_{i}-0, y_{i}-0\right), \quad m>0 .
\end{aligned}
$$

In this paper, motivated by the work above, we will establish the following much more general integral inequality:

$$
\begin{aligned}
u(x, y) \leq & a(x, y)+\sum_{n=1}^{2} \int_{b_{n}\left(x_{0}\right)}^{b_{n}(x)} \int_{c_{n}\left(y_{o}\right)}^{c_{n}(y)} f_{n}(x, y, s, t) \omega_{n}(u(s, t)) d s d t \\
& +g(x, y) \sum_{\left(x_{0}, y_{0}\right)<\left(x_{i}, y_{i}\right)<(x, y)} \beta_{i} u^{m}\left(x_{i}-0, y_{i}-0\right), \quad m>0,
\end{aligned}
$$

with two independent variables involving two nonlinear terms $\omega_{1}(u)$ and $\omega_{2}(u)$ where we do not restrict $\omega_{1}$ and $\omega_{2}$ to the class $\wp$ or the class $J$. Moreover, $f_{n}(x, y, s, t)(n=1,2)$ has a more general form. We also show that many integral inequalities for discontinuous functions such as (1.3)-(1.6) can be reduced to the form of (1.8). Finally, our main result is 
applied to an estimation of the bounds of the solutions of a partial differential equation with impulsive terms.

\section{Main results}

Let

$$
\Omega=\bigcup_{i, j \geq 1} \Omega_{i j}, \quad \Omega_{i j}=\left\{(x, y): x_{i-1} \leq x<x_{i}, y_{j-1} \leq y<y_{j}\right\}
$$

for $i, j=1,2, \ldots, x_{0}>0$ and $y_{0}>0$, and let $D_{1} z(x, y)$ denote the first-order partial derivative of $z(x, y)$ with respect to $x$ and $\sum_{k=0}^{1} u_{k}(x, y)=0$.

Consider (1.8) and assume that

$\left(\mathrm{H}_{1}\right) a(x, y)$ is defined on $\Omega$ and $a\left(x_{0}, y_{0}\right) \neq 0 ; \beta_{i}$ is a nonnegative constant for any positive integer $i$;

$\left(\mathrm{H}_{2}\right) f_{n}(x, y, s, t)(n=1,2)$ are continuous and nonnegative functions on $\Omega \times \Omega$ and satisfy a certain condition: $f_{n}(x, y, s, t)=0(n=1,2)$ if $(s, t) \in \Omega_{i j}, i \neq j$ for arbitrary $i, j=1,2, \ldots$;

$\left(\mathrm{H}_{3}\right) \omega_{1}(u)$ and $\omega_{2}(u)$ are continuous and nonnegative functions on $[0, \infty)$ and are positive on $(0, \infty)$ such that $\frac{\omega_{2}(u)}{\omega_{1}(u)}$ is nondecreasing;

$\left(\mathrm{H}_{4}\right) g(x, y)$ is continuous and nonnegative on $\Omega$;

$\left(\mathrm{H}_{5}\right) u(x, y)$ is nonnegative and continuous on $\Omega$ with the exception of the points $\left(x_{i}, y_{i}\right)$ where there is a finite jump: $u\left(x_{i}-0, y_{i}-0\right) \neq u\left(x_{i}+0, y_{i}+0\right), i=1,2, \ldots$. Here $\left(x_{i}, y_{i}\right)<$ $\left(x_{i+1}, y_{i+1}\right)$ if $x_{i}<x_{i+1}, y_{i}<y_{i+1}, i=0,1,2, \ldots$, and $\lim _{i \rightarrow \infty} x_{i}=\infty, \lim _{i \rightarrow \infty} y_{i}=\infty$;

$\left(\mathrm{H}_{6}\right) b_{n}(x)$ and $c_{n}(y)(n=1,2)$ are continuously differentiable and nondecreasing such that $x_{0} \leq b_{n}(x) \leq x$ on $\left[x_{0}, \infty\right)$ and $y_{0} \leq c_{n}(y) \leq y$ on $\left[y_{0}, \infty\right)$.

Let $W_{j}(u)=\int_{\tilde{u}_{j}}^{u} \frac{d z}{\omega_{j}(z)}$ for $u \geq \tilde{u}_{j}$ and $j=1,2$ where $\tilde{u}_{j}$ is a given positive constant. Clearly, $W_{j}$ is strictly increasing so its inverse $W_{j}^{-1}$ is well defined, continuous, and increasing in its corresponding domain.

Theorem 2.1 Suppose that $\left(\mathrm{H}_{k}\right)(k=1, \ldots, 6)$ hold and $u(x, y)$ satisfies (1.8) for a positive constant m. If we let $u_{i}(x, y)=u(x, y)$ for $(x, y) \in \Omega_{i i}$, then the estimate of $u(x, y)$ is recursively given, for $(x, y) \in \Omega_{i i}, i=1,2, \ldots$, by

$$
\begin{aligned}
u_{i}(x, y) \leq & W_{2}^{-1}\left\{W_{2} \circ W_{1}^{-1}\left[W_{1}\left(r_{i}(x, y)\right)+\int_{b_{1}\left(x_{i-1}\right)}^{b_{1}(x)} \int_{c_{1}\left(y_{i-1}\right)}^{c_{1}(y)} \tilde{f}_{1}(x, y, s, t) d s d t\right]\right. \\
& \left.+\int_{b_{2}\left(x_{i-1}\right)}^{b_{2}(x)} \int_{c_{2}\left(y_{i-1}\right)}^{c_{2}(y)} \tilde{f}_{2}(x, y, s, t) d s d t\right\}
\end{aligned}
$$

where

$$
\begin{aligned}
r_{1}(x, y)= & \max _{x_{0} \leq \xi \leq x, y_{0} \leq \eta \leq y}|a(\xi, \eta)|, \quad \tilde{f}_{n}(x, y, s, t)=\max _{x_{0} \leq \xi \leq x, y_{0} \leq \eta \leq y} f_{n}(\xi, \eta, s, t), \\
r_{i}(x, y)= & r_{1}(x, y)+\sum_{k=1}^{i-1} \sum_{n=1}^{2} \int_{b_{n}\left(x_{k-1}\right)}^{b_{n}\left(x_{k}\right)} \int_{c_{n}\left(y_{k-1}\right)}^{c_{n}\left(y_{k}\right)} f_{n}(x, y, s, t) \omega_{n}\left(u_{k}(s, t)\right) d s d t \\
& +g(x, y) \sum_{k=1}^{i-1} \beta_{k} u_{k}^{m}\left(x_{k}-0, y_{k}-0\right),
\end{aligned}
$$


provided that

$$
\begin{gathered}
W_{1}\left(r_{i}(x, y)\right)+\int_{b_{1}\left(x_{i-1}\right)}^{b_{1}(x)} \int_{c_{1}\left(y_{i-1}\right)}^{c_{1}(y)} \tilde{f}_{1}(x, y, s, t) d s d t \leq \int_{\widetilde{u}_{1}}^{\infty} \frac{d z}{\omega_{1}(z)}, \\
W_{2} \circ W_{1}^{-1}\left[W_{1}\left(r_{i}(x, y)\right)+\int_{b_{1}\left(x_{i-1}\right)}^{b_{1}(x)} \int_{c_{1}\left(y_{i-1}\right)}^{c_{1}(y)} \tilde{f}_{1}(x, y, s, t) d s d t\right] \\
\quad+\int_{b_{2}\left(x_{i-1}\right)}^{b_{2}(x)} \int_{c_{2}\left(y_{i-1}\right)}^{c_{2}(y)} \tilde{f}_{2}(x, y, s, t) d s d t \leq \int_{\tilde{u}_{2}}^{\infty} \frac{d z}{\omega_{2}(z)} .
\end{gathered}
$$

The proof is given in Section 3.

Remark 2.1 If $\omega_{j}$ satisfies $\int_{\tilde{u}_{j}}^{u} \frac{d z}{\omega_{j}(z)}=\infty$ for $j=1,2$, then $i$ in Theorem 2.1 can be any nonzero integer. Reference [4] pointed out that different choices of $\tilde{u}_{j}$ in $W_{j}$ do not affect our results for $j=1,2$. If $a(x, y) \equiv 0$, then define $W_{1}(0)=0$ and (2.1) is still true.

Remark 2.2 If $a(x, y)$ is nondecreasing, Theorem 2.1 generalizes many known results. For example:

(1) If we take $f_{1}(x, y, s, t)=\tau(s, t), f_{2}(x, y, s, t)=0, \omega_{1}(u)=u, m=1, b_{1}(x)=x, c_{1}(y)=y$ and $g(x, y)=1$, then (1.8) reduces to (1.3). It is easy to check that $W_{1}(u)=\ln \frac{u}{\widetilde{u}_{1}}$ and $W_{1}^{-1}(u)=$ $\tilde{u}_{1} e^{u}$. From Theorem 2.1, we know that for $(x, y) \in \Omega_{i i}$

$$
u_{i}(x, y) \leq r_{i}(x, y) e^{\int_{x_{i-1}}^{x} \int_{y_{i-1}}^{y} \tau(s, t) d s d t}
$$

with

$$
r_{i}(x, y)=a(x, y)+\sum_{k=1}^{i-1} \int_{x_{k-1}}^{x_{k}} \int_{y_{k-1}}^{y_{k}} \tau(s, t) u_{k}(s, t) d s d t+\sum_{k=1}^{i-1} \beta_{k} u_{k}\left(x_{k}-0, y_{k}-0\right) .
$$

Hence

$$
\begin{aligned}
& r_{1}(x, y)=a(x, y), \quad u_{1}(x, y)=a(x, y) e^{\int_{x_{0}}^{x} \int_{y_{0}}^{y} \tau(s, t) d s d t}, \\
& r_{2}(x, y)=a(x, y)\left(1+\beta_{1}\right) e^{\int_{x_{0}}^{x_{1}} \int_{y_{0}}^{y_{1}} \tau(s, t) d s d t}, \\
& u_{2}(x, y)=a(x, y)\left(1+\beta_{1}\right) e^{\int_{x_{0}}^{x} \int_{y_{0}}^{y} \tau(s, t) d s d t} .
\end{aligned}
$$

After recursive calculations, we have

$$
u(x, y) \leq a(x, y) \Pi_{\left(x_{0}, y_{0}\right)<\left(x_{i}, y_{i}\right)<(x, y)}\left(1+\beta_{i}\right) e^{\int_{x_{0}}^{x} \int_{y_{0}}^{y} \tau(s, t) d s d t},
$$

which is the same as the expression in [14];

(2) If we take $f_{1}(x, y, s, t)=\tau(s, t), f_{2}(x, y, s, t)=0, \omega_{1}(u)=u, m>0, b_{1}(x)=x, c_{1}(y)=y$ and $g(x, y)=1$, then (1.8) reduces to (1.4) and Theorem 2.1 becomes Theorem 2.1 in [16];

(3) If we take $f_{1}(x, y, s, t)=\tau(s, t), f_{2}(x, y, s, t)=0, \omega_{1}(u)=u^{m}, m>0, b_{1}(x)=x, c_{1}(y)=y$ and $g(x, y)=1$, then (1.8) reduces to (1.5) and Theorem 2.1 becomes Theorem 2.2 in [16]; 
(4) If $\sigma^{\prime}(t)>0$ on $\left[t_{0}, \infty\right)$ where $t_{0}=\min \left\{x_{0}, y_{0}\right\}$, then (1.6) can be rewritten as

$$
\begin{aligned}
u(x, y) \leq & a(x, y)+q(x, y) \int_{\sigma\left(x_{0}\right)}^{\sigma(x)} \int_{\sigma\left(y_{o}\right)}^{\sigma(y)} \frac{\tau\left(\sigma^{-1}(s), \sigma^{-1}(t)\right)}{\sigma^{\prime}\left(\sigma^{-1}(s)\right) \sigma^{\prime}\left(\sigma^{-1}(t)\right)} u^{m}(s, t) d s d t \\
& +\sum_{\left(x_{0}, y_{0}\right)<\left(x_{i}, y_{i}\right)<(x, y)} \beta_{i} u^{m}\left(x_{i}-0, y_{i}-0\right) .
\end{aligned}
$$

If we let $f_{1}(x, y, s, t)=q(x, y) \frac{\tau\left(\sigma^{-1}(s), \sigma^{-1}(t)\right)}{\sigma^{\prime}\left(\sigma^{-1}(s)\right) \sigma^{\prime}\left(\sigma^{-1}(t)\right)}, f_{2}(x, y, s, t)=0$, and $\omega_{1}(u)=u^{m}$, the above inequality is the same as (1.8).

Consider the inequality

$$
\begin{aligned}
\varphi(u(x, y)) \leq & a(x, y)+\sum_{n=1}^{2} \int_{b_{n}\left(x_{0}\right)}^{b_{n}(x)} \int_{c_{n}\left(y_{o}\right)}^{c_{n}(y)} f_{n}(x, y, s, t) \omega_{n}(u(s, t)) d s d t \\
& +g(x, y) \sum_{\left(x_{0}, y_{0}\right)<\left(x_{i}, y_{i}\right)<(x, y)} \beta_{i} \psi\left(u\left(x_{i}-0, y_{i}-0\right)\right),
\end{aligned}
$$

which looks much more complicated than (1.8).

Corollary 2.1 Suppose that $\left(\mathrm{H}_{k}\right)(k=1, \ldots, 6)$ hold, $\psi(u)$ is positive on $(0, \infty), \varphi(u)$ is positive and strictly increasing on $(0, \infty)$ and $u(x, y)$ satisfies $(2.5)$. If we let $u_{i}(x, y)=u(x, y)$ for $(x, y) \in \Omega_{i i}$, then the estimate of $u(x, y)$ is recursively given, for $(x, y) \in \Omega_{i i}, i=1,2, \ldots$, by

$$
\begin{aligned}
u_{i}(x, y) \leq & \varphi^{-1}\left\{W _ { 2 } ^ { - 1 } \left[W_{2} \circ W_{1}^{-1}\left(W_{1}\left(r_{i}(x, y)\right)+\int_{b_{1}\left(x_{i-1}\right)}^{b_{1}(x)} \int_{c_{1}\left(y_{i-1}\right)}^{c_{1}(y)} \tilde{f}_{1}(x, y, s, t) d s d t\right)\right.\right. \\
& \left.\left.+\int_{b_{2}\left(x_{i-1}\right)}^{b_{2}(x)} \int_{c_{2}\left(y_{i-1}\right)}^{c_{2}(y)} \tilde{f}_{2}(x, y, s, t) d s d t\right]\right\},
\end{aligned}
$$

where $W_{j}(u)=\int_{\tilde{u}_{j}}^{u} \frac{d z}{\omega_{j}\left(\varphi^{-1}(z)\right)}, r_{1}(x, y)$ and $\widetilde{f}_{n}(x, y, s, t)$ are given in Theorem $2.1, r_{i}(x, y)$ is defined as follows:

$$
\begin{aligned}
r_{i}(x, y)= & r_{1}(x, y)+\sum_{k=1}^{i-1} \sum_{n=1}^{2} \int_{b_{n}\left(x_{k-1}\right)}^{b_{n}\left(x_{k}\right)} \int_{c_{n}\left(y_{k-1}\right)}^{c_{n}\left(y_{k}\right)} f_{n}(x, y, s, t) \omega_{n}\left(u_{k}(s, t)\right) d s d t \\
& +g(x, y) \sum_{k=1}^{i-1} \beta_{k} \psi\left(u_{k}\left(x_{k}-0, y_{k}-0\right)\right) .
\end{aligned}
$$

Proof Let $\varphi(u(x, y))=h(x, y)$. Since the function $\varphi$ is strictly increasing on $[0, \infty)$, its inverse $\varphi^{-1}$ is well defined. Equation (2.5) becomes

$$
\begin{aligned}
h(x, y) \leq & a(x, y)+\sum_{n=1}^{2} \int_{b_{n}\left(x_{0}\right)}^{b_{n}(x)} \int_{c_{n}\left(y_{o}\right)}^{c_{n}(y)} f_{n}(x, y, s, t) \omega_{n}\left(\varphi^{-1}(h(s, t))\right) d s d t \\
& +g(x, y) \sum_{\left(x_{0}, y_{0}\right)<\left(x_{i}, y_{i}\right)<(x, y)} \beta_{i} \psi\left(\varphi^{-1}\left(h\left(x_{i}-0, y_{i}-0\right)\right)\right) .
\end{aligned}
$$


Let $\widetilde{\omega}_{n}=\omega_{n} \circ \varphi^{-1}$ and $\widetilde{\psi}=\psi \circ \varphi^{-1}$. Equation (2.7) becomes

$$
\begin{aligned}
h(x, y) \leq & a(x, y)+\sum_{n=1}^{2} \int_{b_{n}\left(x_{0}\right)}^{b_{n}(x)} \int_{c_{n}\left(y_{0}\right)}^{c_{n}(y)} f_{n}(x, y, s, t) \widetilde{\omega}_{n}(h(s, t)) d s d t \\
& +g(x, y) \sum_{\left(x_{0}, y_{0}\right)<\left(x_{i}, y_{i}\right)<(x, y)} \beta_{i} \widetilde{\psi}\left(h\left(x_{i}-0, y_{i}-0\right)\right) .
\end{aligned}
$$

It is easy to see that $\widetilde{\psi}(u)>0, \widetilde{\omega}_{1}(u)$ and $\widetilde{\omega}_{2}(u)$ are continuous and nonnegative functions on $[0, \infty)$, and $\frac{\widetilde{\omega}_{2}(u)}{\widetilde{\omega}_{1}(u)}$ is nondecreasing on $(0, \infty)$. Even though $\widetilde{\psi}(u)$ is much more general, in the same way as in Theorem 2.1, for $(x, y) \in \Omega_{i i}, i=1,2, \ldots$, we can obtain the estimate of $u(x, y)$,

$$
\begin{aligned}
u_{i}(x, y) \leq & \varphi^{-1}\left\{W _ { 2 } ^ { - 1 } \left[W_{2} \circ W_{1}^{-1}\left(W_{1}\left(r_{i}(x, y)\right)+\int_{b_{1}\left(x_{i-1}\right)}^{b_{1}(x)} \int_{c_{1}\left(y_{i-1}\right)}^{c_{1}(y)} \tilde{f}_{1}(x, y, s, t) d s d t\right)\right.\right. \\
& \left.\left.+\int_{b_{2}\left(x_{i-1}\right)}^{b_{2}(x)} \int_{c_{2}\left(y_{i-1}\right)}^{c_{2}(y)} \widetilde{f}_{2}(x, y, s, t) d s d t\right]\right\} .
\end{aligned}
$$

This completes the proof of Corollary 2.1.

If $\varphi(u)=u^{\lambda}$ where $\lambda>0$ is a constant, we can study the inequality

$$
\begin{aligned}
u^{\lambda}(x, y) \leq & a(x, y)+\sum_{n=1}^{2} \int_{b_{n}\left(x_{0}\right)}^{b_{n}(x)} \int_{c_{n}\left(y_{o}\right)}^{c_{n}(y)} f_{n}(x, y, s, t) \omega_{n}(u(s, t)) d s d t \\
& +g(x, y) \sum_{\left(x_{0}, y_{0}\right)<\left(x_{i}, y_{i}\right)<(x, y)} \beta_{i} \psi\left(u\left(x_{i}-0, y_{i}-0\right)\right) .
\end{aligned}
$$

According to Corollary 2.1, we have the following result.

Corollary 2.2 Suppose that $\left(\mathrm{H}_{k}\right)(k=1, \ldots, 6)$ hold, $\psi(u)>0$, and $u(x, y)$ satisfies $(2.10)$. If we let $u_{i}(x, y)=u(x, y)$ for $(x, y) \in \Omega_{i i}$, then the estimate of $u(x, y)$ is recursively given, for $(x, y) \in \Omega_{i i}, i=1,2, \ldots$, by

$$
\begin{aligned}
u_{i}(x, y) \leq & \left\{W _ { 2 } ^ { - 1 } \left[W_{2} \circ W_{1}^{-1}\left(W_{1}\left(r_{i}(x, y)\right)+\int_{b_{1}\left(x_{i-1}\right)}^{b_{1}(x)} \int_{c_{1}\left(y_{i-1}\right)}^{c_{1}(y)} \tilde{f}_{1}(x, y, s, t) d s d t\right)\right.\right. \\
& \left.\left.+\int_{b_{2}\left(x_{i-1}\right)}^{b_{2}(x)} \int_{c_{2}\left(y_{i-1}\right)}^{c_{2}(y)} \tilde{f}_{2}(x, y, s, t) d s d t\right]\right\}^{\frac{1}{\lambda}},
\end{aligned}
$$

where $W_{j}(u)=\int_{\widetilde{u}_{j}}^{u} \frac{d z}{\omega\left(z^{\frac{1}{\lambda}}\right)}, r_{1}(x, y), r_{i}(x, y)$ and $\tilde{f}_{n}(x, y, s, t)$ are given in Corollary 2.1 .

\section{Proof of Theorem 2.1}

Obviously, for any $(x, y) \in \Omega, r_{1}(x, y)$ is positive and nondecreasing with respect to $x$ and $y, \tilde{f}_{n}(x, y, s, t)(n=1,2)$ is nonnegative and nondecreasing with respect to $x$ and $y$ for each fixed $s$ and $t$. They satisfy $a(x, y) \leq r_{1}(x, y)$ and $f_{n}(x, y, s, t) \leq \widetilde{f}_{n}(x, y, s, t)$. 
We first consider $(x, y) \in \Omega_{11}=\left\{(x, y): x_{0} \leq x<x_{1}, y_{0} \leq y<y_{1}\right\}$ and have from (1.8)

$$
\begin{aligned}
u(x, y) & \leq a(x, y)+\sum_{n=1}^{2} \int_{b_{n}\left(x_{0}\right)}^{b_{n}(x)} \int_{c_{n}\left(y_{o}\right)}^{c_{n}(y)} f_{n}(x, y, s, t) \omega_{n}(u(s, t)) d s d t \\
& \leq r_{1}(x, y)+\sum_{n=1}^{2} \int_{b_{n}\left(x_{0}\right)}^{b_{n}(x)} \int_{c_{n}\left(y_{o}\right)}^{c_{n}(y)} \tilde{f}_{n}(x, y, s, t) \omega_{n}(u(s, t)) d s d t .
\end{aligned}
$$

Take any fixed $\tilde{x} \in\left(x_{0}, x_{1}\right), \tilde{y} \in\left(y_{0}, y_{1}\right)$, and for arbitrary $x \in\left[x_{0}, \tilde{x}\right], y \in\left[y_{0}, \tilde{y}\right]$ we have

$$
u(x, y) \leq r_{1}(\widetilde{x}, \tilde{y})+\sum_{n=1}^{2} \int_{b_{n}\left(x_{0}\right)}^{b_{n}(x)} \int_{c_{n}\left(y_{0}\right)}^{c_{n}(y)} \widetilde{f}_{n}(\widetilde{x}, \tilde{y}, s, t) \omega_{n}(u(s, t)) d s d t
$$

Let

$$
z(x, y)=r_{1}(\tilde{x}, \tilde{y})+\sum_{n=1}^{2} \int_{b_{n}\left(x_{0}\right)}^{b_{n}(x)} \int_{c_{n}\left(y_{o}\right)}^{c_{n}(y)} \tilde{f}_{n}(\tilde{x}, \tilde{y}, s, t) \omega_{n}(u(s, t)) d s d t
$$

and $z\left(x_{0}, y\right)=r_{1}(\tilde{x}, \tilde{y})$. Hence, $u(x, y) \leq z(x, y)$. Clearly, $z(x, y)$ is a nonnegative, nondecreasing and differentiable function for $x \in\left[x_{0}, \tilde{x}\right]$ and $y \in\left[y_{0}, \tilde{y}\right]$. Moreover, $b_{n}(x)$ (or $c_{n}(y)$ ) is differentiable and nondecreasing in $x \in\left[x_{0}, \tilde{x}\right]$ (or $y \in\left[y_{0}, \tilde{y}\right]$ ) for $n=1,2$. Thus, $b_{n}^{\prime}(x) \geq 0$ (or $c_{n}^{\prime}(y) \geq 0$ ) for $x \in\left[x_{0}, \tilde{x}\right]$ (or $y \in\left[y_{0}, \tilde{y}\right]$ ). Since $r_{1}(\tilde{x}, \tilde{y})>0$ and $\omega_{1}(z(x, y))>0$, we have

$$
\begin{aligned}
\frac{D_{1} z(x, y)}{\omega_{1}(z(x, y))} \leq & \frac{\int_{c_{1}\left(y_{o}\right)}^{c_{1}(y)} \tilde{f}_{1}\left(\tilde{x}, \tilde{y}, b_{1}(x), t\right) b_{1}^{\prime}(x) \omega_{1}\left(u\left(b_{1}(x), t\right)\right) d t}{\omega_{1}(z(x, y))} \\
& +\frac{\int_{c_{2}\left(y_{o}\right)}^{c_{2}(y)} \tilde{f}_{2}\left(\tilde{x}, \tilde{y}, b_{2}(x), t\right) b_{2}^{\prime}(x) \omega_{2}\left(u\left(b_{2}(x), t\right)\right) d t}{\omega_{1}(z(x, y))} \\
\leq & \frac{\int_{c_{1}\left(y_{o}\right)}^{c_{1}(y)} \tilde{f}_{1}\left(\tilde{x}, \tilde{y}, b_{1}(x), t\right) b_{1}^{\prime}(x) \omega_{1}\left(z\left(b_{1}(x), t\right)\right) d t}{\omega_{1}(z(x, y))} \\
& +\frac{\int_{c_{2}\left(y_{o}\right)}^{c_{2}(y)} \tilde{f}_{2}\left(\tilde{x}, \tilde{y}, b_{2}(x), t\right) b_{2}^{\prime}(x) \omega_{2}\left(z\left(b_{2}(x), t\right)\right) d t}{\omega_{1}(z(x, y))} \\
\leq & \frac{\int_{c_{1}\left(y_{o}\right)}^{c_{1}(y)} \tilde{f}_{1}\left(\tilde{x}, \tilde{y}, b_{1}(x), t\right) b_{1}^{\prime}(x) \omega_{1}(z(x, t)) d t}{\omega_{1}(z(x, y))} \\
& +\frac{\int_{c_{2}\left(y_{o}\right)}^{c_{2}(y)} \tilde{f}_{2}\left(\tilde{x}, \tilde{y}, b_{2}(x), t\right) b_{2}^{\prime}(x) \omega_{2}\left(z\left(b_{2}(x), t\right)\right) d t}{\omega_{1}(z(x, y))} \\
\leq & \int_{c_{1}\left(y_{0}\right)}^{c_{1}(y)} \tilde{f}_{1}\left(\tilde{x}, \tilde{y}, b_{1}(x), t\right) b_{1}^{\prime}(x) d t \\
& +\int_{c_{2}\left(y_{0}\right)}^{c_{2}(y)} \tilde{f_{2}}\left(\tilde{x}, \tilde{y}, b_{2}(x), t\right) b_{2}^{\prime}(x) \frac{\omega_{2}\left(z\left(b_{2}(x), t\right)\right)}{\omega_{1}\left(z\left(b_{2}(x), t\right)\right)} d t .
\end{aligned}
$$

Integrating both sides of the above inequality from $x_{0}$ to $x$, we obtain

$$
\begin{aligned}
W_{1}(z(x, y))-W_{1}\left(z\left(x_{0}, y\right)\right) \leq & \int_{x_{0}}^{x} \int_{c_{1}\left(y_{0}\right)}^{c_{1}(y)} \tilde{f}_{1}\left(\tilde{x}, \tilde{y}, b_{1}(s), t\right) b_{1}^{\prime}(s) d s d t \\
& +\int_{x_{0}}^{x} \int_{c_{2}\left(y_{0}\right)}^{c_{2}(y)} \tilde{f}_{2}\left(\tilde{x}, \tilde{y}, b_{2}(s), t\right) b_{2}^{\prime}(s) \frac{\omega_{2}\left(z\left(b_{2}(s), t\right)\right)}{\omega_{1}\left(z\left(b_{2}(s), t\right)\right)} d s d t .
\end{aligned}
$$


Thus,

$$
\begin{aligned}
W_{1}(z(x, y)) \leq & W_{1}\left(r_{1}(\widetilde{x}, \widetilde{y})\right)+\int_{b_{1}\left(x_{0}\right)}^{b_{1}(x)} \int_{c_{1}\left(y_{0}\right)}^{c_{1}(y)} \widetilde{f}_{1}(\widetilde{x}, \tilde{y}, s, t) d s d t \\
& +\int_{b_{2}\left(x_{0}\right)}^{b_{2}(x)} \int_{c_{2}\left(y_{0}\right)}^{c_{2}(y)} \widetilde{f}_{2}(\widetilde{x}, \widetilde{y}, s, t) \phi(z(s, t)) d s d t
\end{aligned}
$$

for $x_{0} \leq x \leq \tilde{x}$ and $y_{0} \leq y \leq \tilde{y}$, where $\phi(u)=\frac{\omega_{2}(u)}{\omega_{1}(u)}$, or equivalently

$$
\begin{aligned}
\xi(x, y) \leq & W_{1}\left(r_{1}(\widetilde{x}, \widetilde{y})\right)+\int_{b_{1}\left(x_{0}\right)}^{b_{1}(x)} \int_{c_{1}\left(y_{0}\right)}^{c_{1}(y)} \tilde{f}_{1}(\tilde{x}, \tilde{y}, s, t) d s d t \\
& +\int_{b_{2}\left(x_{0}\right)}^{b_{2}(x)} \int_{c_{2}\left(y_{0}\right)}^{c_{2}(y)} \tilde{f}_{2}(\widetilde{x}, \widetilde{y}, s, t) \phi\left(W_{1}^{-1}(\xi(s, t))\right) d s d t \triangleq z_{1}(x, y),
\end{aligned}
$$

where

$$
\xi(x, y)=W_{1}(z(x, y)) .
$$

It is easy to check that $\xi(x, y) \leq z_{1}(x, y), z_{1}\left(x_{0}, y\right)=W_{1}\left(r_{1}(\widetilde{x}, \widetilde{y})\right)$ and $z_{1}(x, y)$ is differentiable, positive and nondecreasing on $\left(x_{0}, \widetilde{x}\right]$ and $\left(y_{0}, \widetilde{y}\right]$. Since $\phi\left(W_{1}^{-1}(u)\right)$ is nondecreasing from the assumption $\left(\mathrm{H}_{3}\right)$, we have

$$
\begin{aligned}
& \frac{D_{1} z_{1}(x, y)}{\phi\left(W_{1}^{-1}\left(z_{1}(x, y)\right)\right)} \leq \frac{\int_{c_{1}\left(y_{0}\right)}^{c_{1}(y)} \tilde{f}_{1}\left(\tilde{x}, \tilde{y}, b_{1}(x), t\right) b_{1}^{\prime}(x) d t}{\phi\left(W_{1}^{-1}\left(z_{1}(x, y)\right)\right)} \\
& +\frac{\int_{c_{2}\left(y_{0}\right)}^{c_{2}(y)} \tilde{f}_{2}\left(\widetilde{x}, \tilde{y}, b_{2}(x), t\right) b_{2}^{\prime}(x) \phi\left(W_{1}^{-1}\left(\xi\left(b_{2}(x), t\right)\right)\right) d t}{\phi\left(W_{1}^{-1}\left(z_{1}(x, y)\right)\right)} \\
& \leq \frac{\int_{c_{1}\left(y_{0}\right)}^{c_{1}(x)} \tilde{f}_{1}\left(\widetilde{x}, \tilde{y}, b_{1}(x), t\right) b_{1}^{\prime}(x) d t}{\phi\left[W_{1}^{-1}\left(W_{1}\left(r_{1}(\tilde{x}, \tilde{y})\right)+\int_{b_{1}\left(x_{0}\right)}^{b_{1}(x)} \int_{c_{1}\left(y_{0}\right)}^{c_{1}(y)} \widetilde{f}_{1}(\tilde{x}, \tilde{y}, s, t) d s d t\right)\right]} \\
& +\frac{\int_{\mathcal{c}_{2}\left(y_{0}\right)}^{c_{2}(y)} \tilde{f}_{2}\left(\tilde{x}, \tilde{y}, b_{2}(x), t\right) b_{2}^{\prime}(x) \phi\left(W_{1}^{-1}\left(z_{1}(x, t)\right)\right) d t}{\phi\left(W_{1}^{-1}\left(z_{1}(x, y)\right)\right)} \\
& \leq \frac{\int_{c_{1}\left(y_{0}\right)}^{c_{1}(y)} \tilde{f}_{1}\left(\widetilde{x}, \widetilde{y}, b_{1}(x), t\right) b_{1}^{\prime}(x) d t}{\phi\left[W_{1}^{-1}\left(W_{1}\left(r_{1}(\widetilde{x}, \tilde{y})\right)+\int_{b_{1}\left(x_{0}\right)}^{b_{1}(x)} \int_{c_{1}\left(y_{0}\right)}^{c_{1}(y)} \widetilde{f}_{1}(\widetilde{x}, \tilde{y}, s, t) d s d t\right)\right]} \\
& +\int_{c_{2}\left(y_{0}\right)}^{c_{2}(y)} \tilde{f}_{2}\left(\tilde{x}, \tilde{y}, b_{2}(x), t\right) b_{2}^{\prime}(x) d t \text {. }
\end{aligned}
$$

Note that

$$
\begin{aligned}
\int_{x_{0}}^{x} \frac{D_{1} z_{1}(s, y)}{\phi\left(W_{1}^{-1}\left(z_{1}(s, y)\right)\right)} d s & =\int_{x_{0}}^{x} \frac{D_{1} z_{1}(s, y) \omega_{1}\left(W_{1}^{-1}\left(z_{1}(s, y)\right)\right)}{\omega_{2}\left(W_{1}^{-1}\left(z_{1}(s, y)\right)\right)} d s=\int_{W_{1}^{-1}\left(z_{1}\left(x_{0}, y\right)\right)}^{W_{1}^{-1}\left(z_{1}(x, y)\right)} \frac{d u}{\omega_{2}(u)} \\
& =W_{2} \circ W_{1}^{-1}\left(z_{1}(x, y)\right)-W_{2} \circ W_{1}^{-1}\left(z_{1}\left(x_{0}, y\right)\right) \\
& =W_{2} \circ W_{1}^{-1}\left(z_{1}(x, y)\right)-W_{2} \circ W_{1}^{-1}\left(W_{1}\left(r_{1}(\widetilde{x}, \widetilde{y})\right)\right) \\
& =W_{2} \circ W_{1}^{-1}\left(z_{1}(x, y)\right)-W_{2}\left(r_{1}(\widetilde{x}, \widetilde{y})\right) .
\end{aligned}
$$


Integrating both sides of the inequality (3.6) from $x_{0}$ to $x$, we obtain

$$
\begin{aligned}
& W_{2} \circ W_{1}^{-1}\left(z_{1}(x, y)\right)-W_{2}\left(r_{1}(\tilde{x}, \tilde{y})\right) \\
& =\int_{x_{0}}^{x} \frac{D_{1} z_{1}(s, y)}{\phi\left(W_{1}^{-1}\left(z_{1}(s, y)\right)\right)} d s \\
& \leq \int_{x_{0}}^{x} \frac{\int_{c_{1}\left(y_{0}\right)}^{c_{1}(y)} \tilde{f}_{1}\left(\tilde{x}, \tilde{y}, b_{1}(s), t\right) b_{1}^{\prime}(s) d t}{\phi\left[W_{1}^{-1}\left(W_{1}\left(r_{1}(\tilde{x}, \tilde{y})\right)+\int_{b_{1}\left(x_{0}\right)}^{b_{1}(s)} \int_{c_{1}\left(y_{0}\right)}^{c_{1}(y)} \tilde{f}_{1}(\tilde{x}, \tilde{y}, \tau, t) d \tau d t\right)\right]} d s \\
& +\int_{x_{0}}^{x} \int_{c_{2}\left(y_{0}\right)}^{c_{2}(y)} \widetilde{f}_{2}\left(\tilde{x}, \tilde{y}, b_{2}(s), t\right) b_{2}^{\prime}(s) d s d t \\
& \leq W_{2} \circ W_{1}^{-1}\left[W_{1}\left(r_{1}(\tilde{x}, \widetilde{y})\right)+\int_{b_{1}\left(x_{0}\right)}^{b_{1}(x)} \int_{c_{1}\left(y_{0}\right)}^{c_{1}(y)} \tilde{f}_{1}(\tilde{x}, \tilde{y}, s, t) d s d t\right]-W_{2}\left(r_{1}(\tilde{x}, \widetilde{y})\right) \\
& +\int_{b_{2}\left(x_{0}\right)}^{b_{2}(x)} \int_{c_{2}\left(y_{0}\right)}^{c_{2}(y)} \widetilde{f}_{2}(\tilde{x}, \tilde{y}, s, t) d s d t
\end{aligned}
$$

Thus,

$$
\begin{aligned}
W_{2} \circ W_{1}^{-1}\left(z_{1}(x, y)\right) \leq & W_{2} \circ W_{1}^{-1}\left[W_{1}\left(r_{1}(\tilde{x}, \tilde{y})\right)+\int_{b_{1}\left(x_{0}\right)}^{b_{1}(x)} \int_{c_{1}\left(y_{0}\right)}^{c_{1}(y)} \tilde{f}_{1}(\tilde{x}, \tilde{y}, s, t) d s d t\right] \\
& +\int_{b_{2}\left(x_{0}\right)}^{b_{2}(x)} \int_{c_{2}\left(y_{0}\right)}^{c_{2}(y)} \tilde{f}_{2}(\tilde{x}, \tilde{y}, s, t) d s d t .
\end{aligned}
$$

Hence

$$
\begin{aligned}
u(x, y) \leq & z(x, y) \leq W_{1}^{-1}(\xi(x, y)) \leq W_{1}^{-1}\left(z_{1}(x, y)\right) \\
\leq & W_{2}^{-1}\left[W_{2} \circ W_{1}^{-1}\left(W_{1}\left(r_{1}(\tilde{x}, \tilde{y})\right)+\int_{b_{1}\left(x_{0}\right)}^{b_{1}(x)} \int_{c_{1}\left(y_{0}\right)}^{c_{1}(y)} \widetilde{f}_{1}(\widetilde{x}, \tilde{y}, s, t) d s d t\right)\right. \\
& \left.+\int_{b_{2}\left(x_{0}\right)}^{b_{2}(x)} \int_{c_{2}\left(y_{0}\right)}^{c_{2}(y)} \widetilde{f}_{2}(\tilde{x}, \tilde{y}, s, t) d s d t\right] .
\end{aligned}
$$

Since the above inequality is true for any $x \in\left[x_{0}, \tilde{x}\right], y \in\left[y_{0}, \widetilde{y}\right]$, we obtain

$$
\begin{aligned}
u(\tilde{x}, \tilde{y}) \leq & W_{2}^{-1}\left[W_{2} \circ W_{1}^{-1}\left(W_{1}\left(r_{1}(\tilde{x}, \widetilde{y})\right)+\int_{b_{1}\left(x_{0}\right)}^{b_{1}(\tilde{x})} \int_{c_{1}\left(y_{0}\right)}^{c_{1}(\widetilde{y})} \widetilde{f}_{1}(\widetilde{x}, \tilde{y}, s, t) d s d t\right)\right. \\
& \left.+\int_{b_{2}\left(x_{0}\right)}^{b_{2}(\widetilde{x})} \int_{c_{2}\left(y_{0}\right)}^{c_{2}(\tilde{y})} \widetilde{f}_{2}(\widetilde{x}, \tilde{y}, s, t) d s d t\right] .
\end{aligned}
$$

Replacing $\tilde{x}$ by $x$ and $\tilde{y}$ by $y$ yields

$$
\begin{aligned}
u(x, y) \leq & W_{2}^{-1}\left[W_{2} \circ W_{1}^{-1}\left(W_{1}\left(r_{1}(x, y)\right)+\int_{b_{1}\left(x_{0}\right)}^{b_{1}(x)} \int_{c_{1}\left(y_{0}\right)}^{c_{1}(y)} \widetilde{f}_{1}(x, y, s, t) d s d t\right)\right. \\
& \left.+\int_{b_{2}\left(x_{0}\right)}^{b_{2}(x)} \int_{c_{2}\left(y_{0}\right)}^{c_{2}(y)} \widetilde{f}_{2}(x, y, s, t) d s d t\right] .
\end{aligned}
$$

This means that $(2.1)$ is true for $(x, y) \in \Omega_{11}$ and $i=1$ if replace $u(x, y)$ with $u_{1}(x, y)$. 
For $i=2$ and $(x, y) \in \Omega_{22}=\left\{(x, y): x_{1} \leq x<x_{2}, y_{1} \leq y<y_{2}\right\}$, (1.8) becomes

$$
\begin{aligned}
u(x, y) \leq & r_{1}(x, y)+\sum_{n=1}^{2} \int_{b_{n}\left(x_{0}\right)}^{b_{n}\left(x_{1}\right)} \int_{c_{n}\left(y_{o}\right)}^{c_{n}\left(y_{1}\right)} f_{n}(x, y, s, t) \omega_{n}\left(u_{1}(s, t)\right) d s d t \\
& +g(x, y) \beta_{1} u_{1}^{m}\left(x_{1}-0, y_{1}-0\right) \\
& +\sum_{n=1}^{2} \int_{b_{n}\left(x_{1}\right)}^{b_{n}(x)} \int_{c_{n}\left(y_{1}\right)}^{c_{n}(y)} f_{n}(x, y, s, t) \omega_{n}(u(s, t)) d s d t \\
\leq & r_{2}(x, y)+\sum_{n=1}^{2} \int_{b_{n}\left(x_{1}\right)}^{b_{n}(x)} \int_{c_{n}\left(y_{1}\right)}^{c_{n}(y)} f_{n}(x, y, s, t) \omega_{n}(u(s, t)) d s d t
\end{aligned}
$$

where the definition of $r_{2}(x, y)$ is given in (2.2). Note that the estimate of $u_{1}(x, y)$ is known. Clearly, (3.9) is the same as (3.1) if replace $r_{1}(x, y)$ and $\left(x_{0}, y_{0}\right)$ by $r_{2}(x, y)$ and $\left(x_{1}, y_{1}\right)$. Thus, by (3.8) we have

$$
\begin{aligned}
u(x, y) \leq & W_{2}^{-1}\left[W_{2} \circ W_{1}^{-1}\left(W_{1}\left(r_{2}(x, y)\right)+\int_{b_{1}\left(x_{1}\right)}^{b_{1}(x)} \int_{c_{1}\left(y_{1}\right)}^{c_{1}(y)} \widetilde{f}_{1}(x, y, s, t) d s d t\right)\right. \\
& \left.+\int_{b_{2}\left(x_{1}\right)}^{b_{2}(x)} \int_{c_{2}\left(y_{1}\right)}^{c_{2}(y)} \tilde{f}_{2}(x, y, s, t) d s d t\right] .
\end{aligned}
$$

This implies that (2.1) is true for $(x, y) \in \Omega_{22}$ and $i=2$ if replace $u(x, y)$ by $u_{2}(x, y)$.

Assume that (2.1) is true for $(x, y) \in \Omega_{i i}=\left\{(x, y): x_{i-1} \leq x<x_{i}, y_{i-1} \leq y<y_{i}\right\}$, i.e.,

$$
\begin{aligned}
u_{i}(x, y) \leq & W_{2}^{-1}\left[W_{2} \circ W_{1}^{-1}\left(W_{1}\left(r_{i}(x, y)\right)+\int_{b_{1}\left(x_{i-1}\right)}^{b_{1}(x)} \int_{c_{1}\left(y_{i-1}\right)}^{c_{1}(y)} \widetilde{f}_{1}(x, y, s, t) d s d t\right)\right. \\
& \left.+\int_{b_{2}\left(x_{i-1}\right)}^{b_{2}(x)} \int_{c_{2}\left(y_{i-1}\right)}^{c_{2}(y)} \tilde{f}_{2}(x, y, s, t) d s d t\right] .
\end{aligned}
$$

For $(x, y) \in \Omega_{i+1, i+1}=\left\{(x, y): x_{i} \leq x<x_{i+1}, y_{i} \leq y<y_{i+1}\right\}$, (1.8) becomes

$$
\begin{aligned}
u(x, y) \leq & a(x, y)+\sum_{n=1}^{2} \int_{b_{n}\left(x_{0}\right)}^{b_{n}(x)} \int_{c_{n}\left(y_{0}\right)}^{c_{n}(y)} f_{n}(x, y, s, t) \omega_{n}(u(s, t)) d s d t \\
& +g(x, y) \sum_{\left(x_{0}, y_{0}\right)<\left(x_{i}, y_{i}\right)<(x, y)} \beta_{i} u^{m}\left(x_{i}-0, y_{i}-0\right) \\
\leq & r_{1}(x, y)+\sum_{k=1}^{i} \sum_{n=1}^{2} \int_{b_{n}\left(x_{k-1}\right)}^{b_{n}\left(x_{k}\right)} \int_{c_{n}\left(y_{k-1}\right)}^{c_{n}\left(y_{k}\right)} f_{n}(x, y, s, t) \omega_{n}\left(u_{k}(s, t)\right) d s d t \\
& +g(x, y) \sum_{k=1}^{i} \beta_{k} u_{k}^{m}\left(x_{k}-0, y_{k}-0\right) \\
& +\sum_{n=1}^{2} \int_{b_{n}\left(x_{i}\right)}^{b_{n}(x)} \int_{c_{n}\left(y_{i}\right)}^{c_{n}(y)} f_{n}(x, y, s, t) \omega_{n}(u(s, t)) d s d t \\
\leq & r_{i+1}(x, y)+\sum_{n=1}^{2} \int_{b_{n}\left(x_{i}\right)}^{b_{n}(x)} \int_{c_{n}\left(y_{i}\right)}^{c_{n}(y)} f_{n}(x, y, s, t) \omega_{n}(u(s, t)) d s d t
\end{aligned}
$$


where we use the fact that the estimate of $u(x, y)$ is already known for $(x, y) \in \Omega_{i i}(i=$ $1,2, \ldots)$. Again, (3.11) is the same as (3.1) if replace $r_{1}(x, y)$ and $\left(x_{0}, y_{0}\right)$ by $r_{i+1}(x, y)$ and $\left(x_{i}, y_{i}\right)$. Thus, by (3.8) we have

$$
\begin{aligned}
u(x, y) \leq & W_{2}^{-1}\left[W_{2} \circ W_{1}^{-1}\left(W_{1}\left(r_{i+1}(x, y)\right)+\int_{b_{1}\left(x_{i}\right)}^{b_{1}(x)} \int_{c_{1}\left(y_{i}\right)}^{c_{1}(y)} \tilde{f}_{1}(x, y, s, t) d s d t\right)\right. \\
& \left.+\int_{b_{2}\left(x_{i}\right)}^{b_{2}(x)} \int_{c_{2}\left(y_{i}\right)}^{c_{2}(y)} \tilde{f}_{2}(x, y, s, t) d s d t\right] .
\end{aligned}
$$

This shows that (2.1) is true for $(x, y) \in \Omega_{i+1, i+1}$ if replace $u(x, y)$ by $u_{i+1}(x, y)$. By induction, we know that (2.1) holds for $(x, y) \in \Omega_{i+1, i+1}$ for any nonnegative integer $i$. This completes the proof of Theorem 2.1.

\section{Applications}

Consider the following partial differential equation with an impulsive term:

$$
\left\{\begin{array}{l}
\frac{\partial^{2} v(x, y)}{\partial x \partial y}=H(x, y, v(x, y)), \quad(x, y) \in \Omega_{i i}, x \neq x_{i}, y \neq y_{i}, \\
\left.\triangle v\right|_{x=x_{i}, y=y_{i}}=I_{i}(v), \\
v\left(x, y_{0}\right)=\phi_{1}(x), \quad v\left(x_{0}, y\right)=\phi_{2}(y), \quad \phi_{1}\left(x_{0}\right)=\phi_{2}\left(y_{0}\right) \neq 0,
\end{array}\right.
$$

where $v \in \mathbf{R}, H \in \mathbf{R}, I_{i} \in \mathbf{R}$, and $i=1,2, \ldots$.

Assume that

$\left(C_{1}\right)|H(x, y, v(x, y))| \leq h_{1}(x, y) e^{|v(x, y)|}+h_{2}(x, y) e^{2|v(x, y)|}$ where $h_{1}, h_{2}$ are nonnegative and continuous on $\Omega, h_{1}(x, y)=0, h_{2}(x, y)=0$ for $(x, y) \in \Omega_{i j}, i \neq j, i, j=1,2, \ldots ;$

$\left(C_{2}\right)\left|I_{i}(v)\right| \leq \beta_{i}|v|^{m}$ where $\beta_{i}$ and $m$ are nonnegative constants.

Corollary 4.1 Suppose that $\left(\mathrm{C}_{1}\right)$ and $\left(\mathrm{C}_{2}\right)$ hold. If we let $v_{i}(x, y)=v(x, y)$ for $(x, y) \in \Omega_{i i}$, then the solution of system (4.1) has an estimate for $(x, y) \in \Omega_{i i}$

$$
\begin{aligned}
\left|v_{i}(x, y)\right| \leq & -\frac{1}{2} \ln \left[\left(e^{-r_{i}(x, y)}-\int_{x_{i-1}}^{x} \int_{y_{i-1}}^{y} h_{1}(s, t) d s d t\right)^{2}\right. \\
& \left.-\int_{x_{i-1}}^{x} \int_{y_{i-1}}^{y} h_{2}(s, t) d s d t\right]
\end{aligned}
$$

where

$$
\begin{aligned}
r_{1}(x, y)= & \max _{x_{0} \leq \xi \leq x, y_{0} \leq \eta \leq y}\left|\phi_{1}(\xi)+\phi_{2}(\eta)-\phi_{1}\left(x_{0}\right)\right|>0, \\
r_{i}(x, y)= & r_{1}(x, y)+\sum_{k=1}^{i-1} \int_{x_{k-1}}^{x_{k}} \int_{y_{k-1}}^{y_{k}} h_{1}(s, t) e^{\left|v_{k}(s, t)\right|} d s d t \\
& +\sum_{k=1}^{i-1} \int_{x_{k-1}}^{x_{k}} \int_{y_{k-1}}^{y_{k}} h_{2}(s, t) e^{2\left|v_{k}(s, t)\right|} d s d t+\sum_{k=1}^{i-1} \beta_{k}\left|v_{k}\left(x_{k}-0, y_{k}-0\right)\right|^{m}, \\
\left(e^{-r_{i}(x, y)}\right. & \left.-\int_{x_{i-1}}^{x} \int_{y_{i-1}}^{y} h_{1}(s, t) d s d t\right)^{2}-\int_{x_{i-1}}^{x} \int_{y_{i-1}}^{y} h_{2}(s, t) d s d t>0 .
\end{aligned}
$$


Proof The solution of (4.1) with an initial value is given by

$$
\begin{aligned}
v(x, y)= & v\left(x, y_{0}\right)+v\left(x_{0}, y\right)-v\left(x_{0}, y_{0}\right)+\int_{x_{0}}^{x} \int_{y_{0}}^{y} H(s, t, v(s, t)) d s d t \\
& +\sum_{\left(x_{0}, y_{0}\right)<\left(x_{i}, y_{i}\right)<(x, y)} I_{i}\left(v\left(x_{i}-0, y_{i}-0\right)\right) \\
= & \phi_{1}(x)+\phi_{2}(y)-\phi_{1}\left(x_{0}\right)+\int_{x_{0}}^{x} \int_{y_{0}}^{y} H(s, t, v(s, t)) d s d t \\
& +\sum_{\left(x_{0}, y_{0}\right)<\left(x_{i}, y_{i}\right)<(x, y)} I_{i}\left(v\left(x_{i}-0, y_{i}-0\right)\right),
\end{aligned}
$$

which implies

$$
\begin{aligned}
|v(x, y)| \leq & \left|\phi_{1}(x)+\phi_{2}(y)-\phi_{1}\left(x_{0}\right)\right|+\int_{x_{0}}^{x} \int_{y_{0}}^{y} h_{1}(s, t) e^{|v(s, t)|} d s d t \\
& +\int_{x_{0}}^{x} \int_{y_{0}}^{y} h_{2}(s, t) e^{2|v(s, t)|} d s d t+\sum_{\left(x_{0}, y_{0}\right)<\left(x_{i}, y_{i}\right)<(x, y)} \beta_{i}\left|v\left(x_{i}-0, y_{i}-0\right)\right|^{m} .
\end{aligned}
$$

Let

$$
\begin{aligned}
& u(x, y)=|v(x, y)|, \quad a(x, y)=\left|\phi_{1}(x)+\phi_{2}(y)-\phi_{1}\left(x_{0}\right)\right|, \quad b_{1}(x)=b_{2}(x)=x, \\
& c_{1}(y)=c_{2}(y)=y, \quad g(x, y)=1, \quad \omega_{1}(u)=e^{u}, \quad \omega_{2}(u)=e^{2 u}, \\
& f_{1}(x, y, s, t)=h_{1}(s, t), \quad f_{2}(x, y, s, t)=h_{2}(s, t) .
\end{aligned}
$$

Thus, (4.4) is the same as (1.8). It is easy to see that for any positive constants $\tilde{u}_{1}$ and $\tilde{u}_{2}$

$$
\begin{aligned}
& r_{1}(x, y)=\max _{x_{0} \leq \xi \leq x, y_{0} \leq \eta \leq y}|a(\xi, \eta)|>0, \\
& \tilde{f}_{1}(x, y, s, t)=h_{1}(s, t), \quad \widetilde{f}_{2}(x, y, s, t)=h_{2}(s, t), \\
& W_{1}(u)=\int_{\widetilde{u}_{1}}^{u} \frac{d z}{\omega_{1}(z)}=\int_{\widetilde{u}_{1}}^{u} e^{-z} d z=e^{-\widetilde{u}_{1}}-e^{-u}, \quad W_{1}^{-1}(u)=-\ln \left(e^{-\widetilde{u}_{1}}-u\right), \\
& W_{2}(u)=\int_{\widetilde{u}_{2}}^{u} \frac{d z}{\omega_{2}(z)}=\int_{\widetilde{u}_{2}}^{u} e^{-2 z} d z=\frac{1}{2}\left(e^{-2 \widetilde{u}_{2}}-e^{-2 u}\right), \\
& W_{2}^{-1}(u)=-\frac{1}{2} \ln \left(e^{-2 \widetilde{u}_{2}}-2 u\right), \\
& r_{i}(x, y)= \\
& r_{1}(x, y)+\sum_{k=1}^{i-1} \int_{x_{k-1}}^{x_{k}} \int_{y_{k-1}}^{y_{k}} h_{1}(s, t) e^{\left|v_{k}(s, t)\right|} d s d t \\
& \quad+\sum_{k=1}^{i-1} \int_{x_{k-1}}^{x_{k}} \int_{y_{k-1}}^{y_{k}} h_{2}(s, t) e^{2\left|v_{k}(s, t)\right|} d s d t+\sum_{k=1}^{i-1} \beta_{k}\left|v_{k}\left(x_{k}-0, y_{k}-0\right)\right|^{m} .
\end{aligned}
$$

Therefore, for any nonnegative $i$ and $(x, y) \in \Omega_{i i}$

$$
\left|v_{i}(x, y)\right| \leq-\frac{1}{2} \ln \left[\left(e^{-r_{i}(x, y)}-\int_{x_{i-1}}^{x} \int_{y_{i-1}}^{y} h_{1}(s, t) d s d t\right)^{2}-\int_{x_{i-1}}^{x} \int_{y_{i-1}}^{y} h_{2}(s, t) d s d t\right]
$$


provided that

$$
\left(e^{-r_{i}(x, y)}-\int_{x_{i-1}}^{x} \int_{y_{i-1}}^{y} h_{1}(s, t) d s d t\right)^{2}-\int_{x_{i-1}}^{x} \int_{y_{i-1}}^{y} h_{2}(s, t) d s d t>0 .
$$

Remark 4.1 From (4.4), we know $\omega_{1}(u)=e^{u}$. Clearly, $\omega_{1}(2 u)=e^{2 u} \leq \omega_{1}(2) \omega_{1}(u)=e^{2} e^{u}$ does not hold for large $u>0$. Thus, $\omega_{1}(u)=e^{u}$ does not belong to the class $\wp$ in [24]. Again $\omega_{1}\left(\frac{u}{2}\right)=e^{\frac{u}{2}} \geq \frac{1}{2} \omega_{1}(u)=\frac{1}{2} e^{u}$ does not hold for large $u>0$ so $\omega_{1}(u)$ does not belong to the class $J$ in [24]. Hence, the results in [24] cannot be applied to the inequality (4.1).

\section{Competing interests}

The author declares that she has no competing interests.

\section{Acknowledgements}

The author is grateful to Dr. Shengfu Deng for many helpful conversations during the course of this work. This research was supported by National Natural Science Foundation of China (No. 11371314), Guangdong Natural Science Foundation (No. S2013010015957), and the Project of Department of Education of Guangdong Province, China (No. 2012KJCX0074)

Received: 29 October 2013 Accepted: 8 May 2014 Published: 15 December 2014

\section{References}

1. Agarwal, RP: On an integral inequality in $n$ independent variables. J. Math. Anal. Appl. 85, 192-196 (1982)

2. Agarwal, RP, Deng, SF, Zhang, WN: Generalization of a retard Gronwall-like inequality and its applications. Appl. Math. Comput. 165, 599-612 (2005)

3. Bihari, IA: Generalization of a lemma of Bellman and its application to uniqueness problems of differential equations. Acta Math. Hung. 7, 81-94 (1956)

4. Choi, SK, Deng, SF, Koo, NJ, Zhang, WN: Nonlinear integral inequalities of Bihari-type without class H. Math. Inequal. Appl. 8, 643-654 (2005)

5. Cheung, W, lovane, G: Some new nonlinear inequalities and applications to boundary value problems. Nonlinear Anal. 64, 2112-2128 (2006)

6. Rakhmatullina, LF: On application of solvability conditions of Chaplygin problems of boundedness and solution stability of differential equations. Izv. Vysš. Učebn. Zaved., Mat. 2, 198-201 (1959)

7. Sun, YG: On retarded integral inequalities and their applications. J. Math. Anal. Appl. 301, 265-275 (2005)

8. $\mathrm{Wu}, \mathrm{Y}, \mathrm{Li}, \mathrm{XP}$, Deng, SF: Nonlinear delay discrete inequalities and their applications to Volterra type difference equations. Adv. Differ. Equ. 2010, Artcle ID 795145 (2010)

9. Zheng, KL, Zhong, SM: Nonlinear sum-difference inequalities with two variables. Int. J. Appl. Math. Comput. Sci. 6 , 31-36 (2010)

10. Wang, WS: A generalized retarded Gronwall-like inequality in two variable and applications to BVP. Appl. Math. Comput. 191, 144-154 (2007)

11. Zhang, WN, Deng, SF: Projected Gronwall-Bellman's inequality for integrable functions. Math. Comput. Model. 34 394-402 (2001)

12. Angela, G, Anna, MP: On some generalizations Bellman-Bihari result for integro-functional inequalities for discontinuous functions and their applications. Bound. Value Probl. 2009, Article ID 808124 (2009)

13. Borysenko, SD: About asymptotical stability on linear approximation of the systems with impulse influence. Ukr. Mat. Zh. 35, 144-150 (1982)

14. Borysenko, SD: Integro-sum inequalities for functions of many independent variables. Differ. Equ. 25, 1634-1641 (1989)

15. Borysenko, SD, Ciarletta, M, lovane, G: Integro-sum inequalities and motion stability of systems with impulse perturbations. Nonlinear Anal. 62, 417-428 (2005)

16. Borysenko, SD, lovane, G: About some new integral inequalities of Wendroff type for discontinuous functions Nonlinear Anal. 66, 2190-2203 (2007)

17. Borysenko, SD, lovane, G, Giordano, P: Investigations of properties motion for essential nonlinear systems perturbed by impulses on some hypersurfaces. Nonlinear Anal. 62, 345-363 (2005)

18. Borysenko, SD, Toscano, S: Impulsive differential system: the problem of stability and practical stability. Nonlinear Anal. 71, e1843-e1849 (2009)

19. Gallo, A, Piccirllo, AM: On some generalizations Bellman-Bihari result for integrofunctionam inequalities for discontinuous functions and theri applications. Bound. Value Probl. 2009, Article ID 808124 (2009)

20. Gallo, A, Piccirllo, AM: About new analogies of Gronwall-Bellman-Bihari type inequalities for discontinuous functions and estimated solution for impulsive differential systems. Nonlinear Anal. 67, 1550-1568 (2007)

21. Iovane, G: Some new integral inequalities of Bellman-Bihari type with delay for discontinuous functions. Nonlinear Anal. 66, 498-508 (2007)

22. lovane, G: On Gronwall-Bellman-Bihari type integral inequalities in several variables with retardation for discontinuous functions. Math. Inequal. Appl. 11, 599-606 (2008)

23. Mitropolskiy, YA, lovane, G, Borysenko, SD: About a generalization of Bellman-Bihari type inequalities for discontinuous functions and theri applications. Nonlinear Anal. 66, 2140-2165 (2007) 
24. Gallo, A, Piccirillo, AM: Multidimensional impulse inequalities and general Bihari-type inequalities for discontinuous functions with delay. Nonlinear Stud. 19, 115-126 (2012)

25. Samoilenko, AM, Perestjuk, NA: Stability of the solutions of differential equations with impulsive action. Appl. Math. Comput. 165, 599-612 (2005)

26. Samoilenko, AM, Perestyuk, NA: Differential Equations with Impulse Effect. Visha Shkola, Kyiv (1987)

27. Samoilenko, AM, Perestyuk, NA: Stability of the solutions of differential equations with impluse action. Appl. Math. Comput. 195, 599-613 (2005)

doi:10.1186/1029-242X-2014-524

Cite this article as: Mi: Generalized integral inequalities for discontinuous functions with two independent variables and their applications. Journal of Inequalities and Applications 2014 2014:524.

Submit your manuscript to a SpringerOpen ${ }^{\circ}$ journal and benefit from:

- Convenient online submission

- Rigorous peer review

- Immediate publication on acceptance

- Open access: articles freely available online

- High visibility within the field

- Retaining the copyright to your article

Submit your next manuscript at $>$ springeropen.com 